\title{
Incidence and risk factors for early readmission after hospitalization for SARS-CoV-2 infection: results from a retrospective cohort study
}

\author{
Cristina Kirkegaard ${ }^{1} \cdot$ Anna Falcó-Roget $^{1}$ - Adrián Sánchez-Montalvá ${ }^{1,2} \cdot$ Ángel Valls $^{3} \cdot$ David Clofent $^{4}$. \\ Isabel Campos-Varela ${ }^{5}$. Sonia García-García ${ }^{6}$. Lina María Leguízamo ${ }^{7} \cdot$ Júlia Sellarès-Nadal $^{1} \cdot$ Simeon Eremiev $^{1}$. \\ Miguel Villamarín ${ }^{1} \cdot$ Blanca Marzo ${ }^{1} \cdot$ Benito Almirante ${ }^{1,8} \cdot$ Òscar Len $^{1,8}$ (B)
}

Received: 10 May 2021 / Accepted: 5 July 2021 / Published online: 30 July 2021

๑) Springer-Verlag GmbH Germany, part of Springer Nature 2021

\begin{abstract}
Purpose We aim to assess risk factors related to early readmission in previous hospitalized patients with COVID-19. Methods We analyzed a retrospective cohort of patients with laboratory-confirmed COVID-19 admitted to Vall d'Hebron University Hospital, Barcelona, Spain. Early readmission was defined as the need for hospitalization within a period of 60 days after discharge. A descriptive analysis of the readmission was performed, including hospitalization outcome. We also performed a multivariate logistic regression to define risk factors for readmission

Results A total of 629 patients were followed up during 60 days with a readmission cumulative incidence of 5.4\% (34 out of 629) and an incidence rate of 0.034 person-years. Main reasons for readmission were respiratory worsening $(13,38.2 \%)$, decompensation of previous disease $(12,35.3 \%)$ or infectious complications $(6,17.6 \%)$. Median time to readmission was 12 days (interquartile range 7-33 days). Prior diagnosis of heart failure (OR 4.09; 95\% CI 1.35-12.46; $p=0.013$ ), length of stay during index admission greater than 13 days (OR 2.72; 95\% CI 1.21-6.12; $p=0.015$ ), treatment with corticosteroids (OR 2.39; 95\% CI 1.01-5.70; $p=0.049$ ) and developing pulmonary thromboembolism (OR 11.59; 95\% CI 2.89-46.48; $p=0.001$ ) were the risk factors statistically associated with early readmission.

Conclusion Readmission cumulative incidence was 5.4\%. Those patients with prior diagnosis of heart failure, length of stay greater than 13 days, treated with corticosteroids or who developed pulmonary thromboembolism might benefit from close monitoring after being discharged.
\end{abstract}

Keywords SARS-CoV-2 $\cdot$ Readmission $\cdot$ Risk factors

Cristina Kirkegaard and Anna Falcó-Roget authors contributed equally to the work.

Òscar Len

oscar.len@vhir.org

1 Infectious Diseases Department, University Hospital Vall d'Hebron, Universitat Autónoma de Barcelona, Barcelona, Spain

2 Programa de Salut Internacional de Catalunya del Institut Català de la Salut (PROSICS), Barcelona, Spain

3 Internal Medicine Department, University Hospital Vall d'Hebron, Universitat Autónoma de Barcelona, Barcelona, Spain

4 Pneumology Department, University Hospital Vall d'Hebron, Universitat Autónoma de Barcelona, Barcelona, Spain
5 Internal Medicine-Hepatology Department, University Hospital Vall d'Hebron, Universitat Autónoma de Barcelona, Barcelona, Spain

6 Pharmacy Department, University Hospital Vall d'Hebron, Universitat Autónoma de Barcelona, Barcelona, Spain

7 Clinical Pharmacology Department, University Hospital Vall d'Hebron, Universitat Autónoma de Barcelona, Barcelona, Spain

8 Spanish Network for the Study of Infectious Diseases (REIPI), Barcelona, Spain 


\section{Introduction}

The ongoing coronavirus disease pandemic (COVID-19) caused by severe acute respiratory syndrome coronavirus 2 (SARS-CoV-2) was first described in Wuhan, China, and rapidly progressed worldwide affecting more than 179 million people and causing more than 3.8 million deaths [1].

The spectrum of this disease goes from asymptomatic patients to severe pneumonia. In this last group, some patients develop rapidly progressing respiratory failure and acute respiratory distress syndrome (ARDS), multi-organ and systemic manifestations and/or complications during hospitalization, such as heart failure, acute kidney failure, liver dysfunction, deep vein thrombosis, pulmonary thromboembolism (PTE), superinfection, arrhythmia or stroke [2, 3].

Previous researches have analyzed the data of COVID-19 patients to describe risk factors of mortality. Older age, high number of comorbidities, higher levels of acute-phase reactants, such as C-reactive protein (CRP), procalcitonin (PCT) and D-dimer, were associated with more severity of infection [4-6]. Unfortunately, limited studies have paid attention to the risk factors related to readmission after being discharged [7-11].

In this context, we aim to describe the characteristics of hospitalized patients with COVID-19 pneumonia who were readmitted early after discharge and determine risk factors of readmission.

\section{Methods}

\section{Study setting and population}

The Vall d'Hebron COVID-19 Cohort Study includes all consecutive adult patients ( $\geq 18$ years old) admitted for COVID-19 at Vall d'Hebron University Hospital, Barcelona, Spain. Between March 1, 2020 and May 31, 2020, data were collected from 870 patients. The presence of SARS-CoV-2 was determined by RT-PCR performed on any respiratory sample (nasopharyngeal swab, sputum, bronchoalveolar lavage or aspirate, tracheal aspirate).

The standard of care in our hospital consisted of the first wave of lopinavir/ritonavir or darunavir/cobicistat according to underlying diseases and tolerance, hydroxychloroquine and azithromycin. At the beginning, azithromycin was administered as an antibiotic but later it was used as an immunomodulatory agent. Antimicrobial prescription depended on clinician criteria.

\section{Study objectives}

The primary objective was to determine the risk factors associated with the readmission. Secondary objectives were establishing the cumulative incidence and defining the reasons for readmission.

\section{Data collection}

Data were collected in a dedicated web-based individual password-protected database with real-time data quality rules and data traceability system hosted in REdCAP. The following information was retrieved from the database: demographic characteristics, baseline diseases and past medical history (arterial hypertension, diabetes mellitus, chronic kidney disease, pulmonary disease, liver disease, heart failure, history of cancer, autoimmune disease, immunosuppression, hematologic disease and obesity), Charlson Comorbidity Index and analytical, clinical, microbiological and radiological data from the acute infection and its evolution. Readmission information was collected retrospectively from the electronic health record system of the patients.

\section{Definitions}

Early readmission was determined as the need for hospitalization by any cause within a period of 60 days after the initial COVID-19 episode. Readmission was assessed in the network of public hospitals of the Catalan Institute of Health and the private hospitals of the metropolitan area of Barcelona. Readmissions occurring in others outside Catalonia were missed. Those patients who only attended the emergency department were excluded. Patients lost to follow-up were those who did not have contact with any health facility for 60 days or more after being discharged.

Treatment with corticoids was defined as a dose greater than $0.5 \mathrm{mg} / \mathrm{kg}$ per day of prednisone (or equivalent) for at least 3 days during admission. Regarding complications, ARDS was defined as an acute beginning and a $P / F$ ratio $<200$ or $\mathrm{SF}$ ratio $<235$ (equals the arterial $\mathrm{pO}_{2}$ or oxygen saturation, respectively, divided by the fraction of inspired oxygen expressed as a decimal) with bilateral infiltrates in the chest radiography and clinical evidence of left auricular hypertension. Respiratory failure was defined as a $\mathrm{pO}_{2}<60 \mathrm{mmHg}$, an oxygen saturation $<90 \%$, a $P / F$ ratio $<300$ or a $\mathrm{SF}$ ratio $<315$. Septic shock was designated as a need of vasoactive drugs to maintain a mean arterial pressure greater than $65 \mathrm{mmHg}$ and hyperlactatemia ( $>2 \mathrm{mmol} / \mathrm{L}$ ) in absence of hypovolemia. Acute renal failure was characterized as an increase in serum creatinine by $\geq 1.5$ times baseline or a fall of $25 \%$ of glomerular filtration rate 
and liver dysfunction referred to an absolute increase in serum transaminases by $\geq 3$ times baseline or gamma-glutamyl transferase (GGT) and alkaline phosphatase (ALP) by $\geq 5$. Finally, a secondary infection was described as another infection different from SARS-CoV-2.

\section{Statistical analysis}

Continuous variables are presented as mean and standard deviation while those variables that do not follow a normal distribution are presented as median and interquartile range. Both were compared using Student's $t$ test or Mann-Whitney $U$ Test, respectively. Categorical variables are presented as $N$ and percentage (\%) and were compared using Chi-square or Fisher's exact test. Receiver operating characteristic curve (ROC) analysis was performed to determine cut-off values for continuous variables.

Uni- and multivariate odds ratios (OR) were calculated using multiple logistic regression analysis to identify risk factors. Variables showing statistically significant differences between patients with or without readmission in the univariate analysis were then tested in multivariate models. Models were performed in a sequential fashion beginning with the variable most strongly associated with readmission. In addition, clinically relevant factors with $p$ values $<0.1$ that were considered to be potential confounders based on experience and data in the literature were forced into the multivariate model to investigate their effect.

The threshold of statistical significance was $p \leq 0.05$. The statistical analysis was performed using IBM SPSS Statistics for Windows, Version 25.0. Armonk, NY: IBM Corp. Released 2017.

\section{Results}

\section{Patients}

Flow diagram for patient selection is shown in Fig. 1. The readmission cumulative incidence was $5.4 \%$. Among readmitted patients, one patient died during the second hospitalization episode. The median time for readmission was 12 days (IQR 7-33 days) with half of the 60-day readmissions occurring within 2 weeks of discharge. Thirteen out of $34(38.2 \%)$ were secondary to respiratory worsening, 12 patients (35.3\%) related to a decompensation of a known underlying disease, and six (17.6\%) due to infectious complications. Three patients were readmitted because of pregnancy related events, one was a post-cesarean wound complication, and the other two were not considered a complication of acute COVID-19 (one term labor, one hyperemesis). Diagnosis made during the readmission and days until readmission are detailed in Table 1 and Fig. 2.

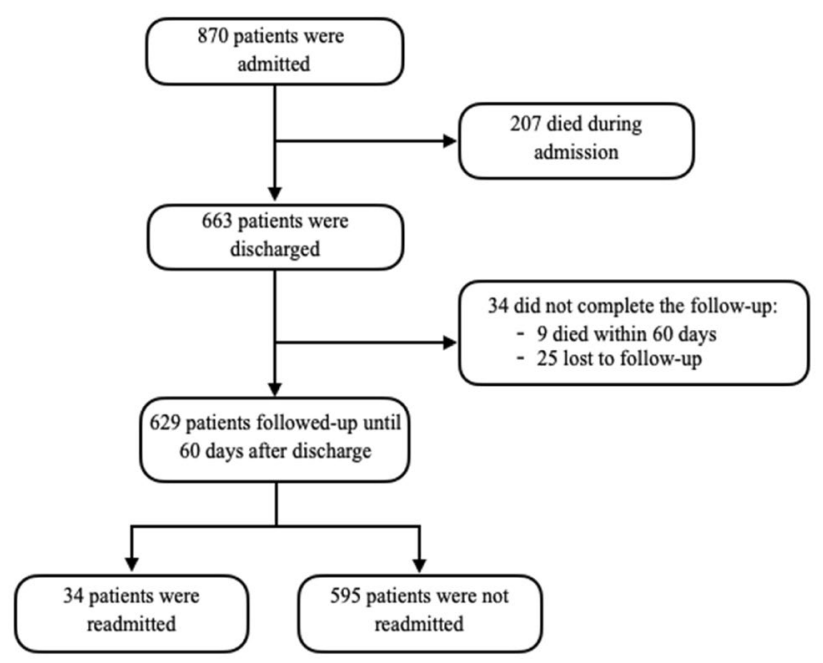

Fig. 1 Study cohort and patients' selection

\section{Patients' baseline characteristics}

The mean age of patients was approximately 60 years and $50.6 \%$ were male. The main comorbidities were arterial hypertension $(40.4 \%)$, obesity $(18.6 \%)$, diabetes $(18.1 \%)$ and pulmonary disease (17\%). The main epidemiological and clinical data of included patients are shown in Table 2.

Among patients' characteristics, male gender $(p=0.040)$ and both smoking $(p=0.013)$ and alcohol abuse $(p=0.004)$ were significantly different among groups. Readmitted patients were also more likely to have a previous diagnosis of cancer $(p=0.025)$, heart failure $(p=0.001)$ and Charlson Comorbidity Index greater than 3 (CCI; $p=0.003$ ).

Finally, the median length of stay during index admission was 9 days. An increased length of stay was observed in those patients who required re-hospitalization (20 days versus 9 days, $p<0.001)$.

\section{Laboratory and radiological characteristics}

Laboratory and radiological findings are detailed in Tables 3 and 4 , respectively.

Readmitted patients were more likely to present lower absolute lymphocyte count $(p=0.014)$ and higher levels of D-dimer $(p=0.014)$. No other statistically significant differences were found amongst the other variables.

Radiological abnormalities were seen in 534 hospitalized patients (84.9\%) and included the presence of focal consolidation, ground glass opacities, pleural effusion and/other abnormalities. Readmitted patients had a lower percentage $(57.9 \%)$ than that of non-readmitted patients $(86.6 \%)$ regarding pulmonary affectation $(p<0.001)$. We distinguished between unilateral or bilateral affectation; we found no significant difference between the two groups. On the other 
Table 1 Diagnosis of readmitted patients and days until readmission after hospitalization for SARS-CoV-2 infection

\begin{tabular}{lll}
\hline & Cases & Diagnosis (days until readmission) \\
\hline Related with pregnancy 3 (8.8) & 1 & Hyperemesis gravidarum (2) \\
& 1 & Term labor (48) \\
Respiratory worsening 13 (38.2) & 1 & Surgical wound dehiscence post-cesarean Section (7) \\
& 1 & Pulmonary thromboembolism $(3,7,7)$ \\
& 4 & Organizing pneumonia (17) \\
& 1 & Hypoxic respiratory failure (2, 1, 1, 4) \\
& 1 & Tracheal granuloma after endotracheal intubation (44) \\
Decompensation of an underlying & 2 & Thoracic pain (18, 8) \\
& 1 & Self-limited febrile syndrome (9) \\
& 2 & Cirrhosis decompensation (49, 35) \\
& 2 & Bile duct pathology (9, 39) \\
& 1 & Bronchoaspiration due to dysphagia (60) \\
& 4 & Acute heart failure (4, 21, 39, 8) \\
& 1 & Percutaneous endoscopic gastrostomy dysfunction (11) \\
& 1 & Esophageal stent migration (19) \\
& 1 & Pacemaker mediated atrial tachycardia (13) \\
& 2 & Infection caused by Clostridiodes difficile (25, 32) \\
& 1 & Fever due to teicoplanin (11) \\
& 1 & Acute appendicitis (10) \\
& 1 & Herpetic stomatitis (18) \\
& 1 & Pneumonia caused by Pseudomonas aeruginosa (25) \\
\hline
\end{tabular}

*Data are expressed as numbers and percentages unless otherwise indicated

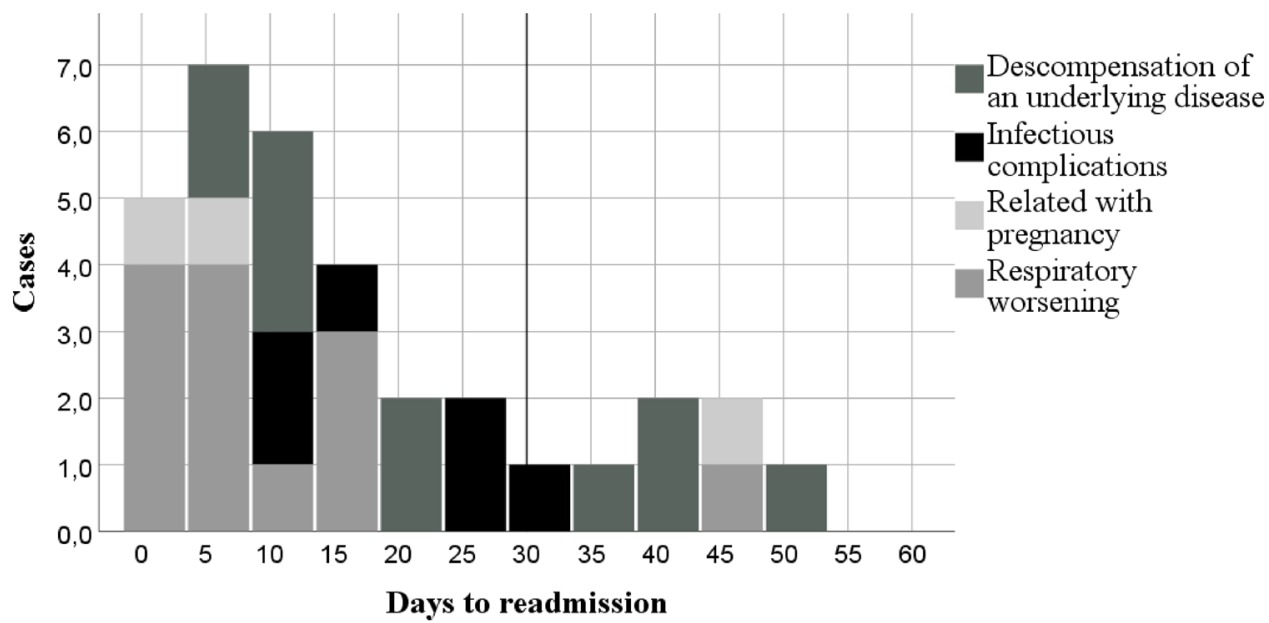

hand, more patients presented with radiological affectation greater than $33 \%$ in the readmitted group $(11 / 22,50 \%$ versus $140 / 512,27.3 \% ; p=0.007)$.

\section{Treatment}

The treatment administered to patients is detailed in Table 5.

Seventy-two patients $(11.4 \%)$ received corticosteroids during hospitalization and ten were readmitted $(29.4 \%)$. Higher readmission rates were observed among those patients who received corticosteroids during their first admission $(p=0.001)$. Patients with higher levels of IL-6 (greater than $40 \mathrm{pg} / \mathrm{L}$ ) could receive tocilizumab as a rescue treatment. Although these patients had higher rates of readmission $(26.5 \%$ versus $17.1 \%)$, there were no statistically significant differences between both groups.

Non-readmitted patients received prophylactic anticoagulation in higher percentages than that of readmitted patients $(69.9 \%$ versus $48.5 \%, p<0.001)$. Prophylactic 
Table 2 Epidemiological and clinical data from patients discharged after hospitalization for SARS-CoV-2 infection
Table 3 Laboratory findings from patients discharged after hospitalization for SARS-CoV-2 infection

\begin{tabular}{llllr}
\hline & Global $(N=629)$ & Readmitted $(N=34)$ & $\begin{array}{l}\text { Non-readmit- } \\
\text { ted }(N=595)\end{array}$ & $P$ value \\
\hline Age $($ mean \pm SD) & $60.28 \pm 17.37$ & $62.59 \pm 20.23$ & $60.42 \pm 17.2$ & 0.479 \\
Male & $318(50.6)$ & $23(67.6)$ & $295(49.6)$ & $\mathbf{0 . 0 4 0}$ \\
Sometime smoker & $163(25.9)$ & $15(44.1)$ & $148(24.9)$ & $\mathbf{0 . 0 1 3}$ \\
Sometime drinker & $29(4.6)$ & $5(14.7)$ & $24(4.0)$ & $\mathbf{0 . 0 0 4}$ \\
Diabetes & $114(18.1)$ & $10(29.4)$ & $104(17.5)$ & 0.079 \\
Immunosuppression & $53(8.4)$ & $5(14.7)$ & $48(8.1)$ & 0.175 \\
Autoimmune disease & $6(1)$ & $0(0)$ & $6(1)$ & 1.000 \\
Cancer diagnosis & $60(9.6)$ & $7(20.6)$ & $53(8.9)$ & $\mathbf{0 . 0 2 5}$ \\
Hematologic disease & $24(3.8)$ & $1(2.9)$ & $23(3.9)$ & 1.000 \\
Arterial hypertension & $254(40.4)$ & $16(47.1)$ & $238(40)$ & 0.415 \\
Heart-failure & $26(4.1)$ & $5(14.7)$ & $21(3.5)$ & $\mathbf{0 . 0 0 1}$ \\
Asthma & $31(4.9)$ & $3(8.8)$ & $28(4.7)$ & 0.231 \\
COPD & $30(4.8)$ & $2(5.9)$ & $28(4.7)$ & 0.673 \\
Interstitial lung disease & $46(7.3)$ & $4(11.8)$ & $42(7.1)$ & 0.302 \\
Chronic kidney disease & $65(10.3)$ & $3(8.8)$ & $62(10.4)$ & 1.000 \\
Dialysis & $10(1.6)$ & $0(0)$ & $10(1.7)$ & 1.000 \\
Liver disease & $24(3.8)$ & $2(5.9)$ & $22(3.7)$ & 0.377 \\
Obesity & $117(18.6)$ & $5(14.7)$ & $112(18.8)$ & 0.548 \\
CCI $>3$ & $29.0(181)$ & $17(51.5)$ & $164(27.7)$ & $\mathbf{0 . 0 0 3}$ \\
Previous treatment with ACEI & $88(14)$ & $4(11.8)$ & $84(14.1)$ & 1.000 \\
Previous treatment with ARB & $66(10.5)$ & $4(11.8)$ & $62(10.4)$ & 0.773 \\
Previous treatment with NSAID & $24(3.8)$ & $2(5.9)$ & $22(3.7)$ & 0.377 \\
Length of stay (days, median, IQR) & $9(6-19)$ & $20(9-36)$ & $9(9-18)$ & $<.001$ \\
\hline Bold & & & & \\
\hline
\end{tabular}

Bold values indicate statistically significant

COPD Chronic Obstructive Pulmonary Disease; CCI Charlson Comorbidity Index; ACEI AngiotensinConverting-Enzyme Inhibitors; ARB Angiotensin-II receptor blockers; NSAID Non-Steroidal Anti-Inflammatory Drug

*Data are expressed as number and percentages unless otherwise indicated

\begin{tabular}{lcccc}
\hline & Global $(N=629)$ & Readmitted $(N=34)$ & Non-readmitted $(N=595)$ & $P$ value \\
\hline Leucocytes & $6.21(4.72-8.00)$ & $6.98(4.77-9.06)$ & $6.18(4.72-7.92)$ & 0.192 \\
ANC & $4.43(3.11-6.26)$ & $4.93(3.14-7.15)$ & $4.44(3.11-6.11)$ & 0.253 \\
ALC & $1.01(0.76-1.40)$ & $0.83(0.57-1.23)$ & $1.02(0.77-1.40)$ & $\mathbf{0 . 0 1 8}$ \\
Fibrinogen & $4.98(4.42-5.76)$ & $4.89(4.31-5.35)$ & $4.99(4.42-5.77)$ & 0.197 \\
D-dimer & $252.00(168.00-436.00)$ & $313(237-1153.75)$ & $247(166-425)$ & $\mathbf{0 . 0 1 4}$ \\
LDH & $316.00(264.00-412.75)$ & $284(214.5-494.5)$ & $318(265-412)$ & 0.362 \\
C-reactive protein & $8.08(3.52-15.99)$ & $10.79(3.68-17.54)$ & $8.01(3.50-15.92)$ & 0.757 \\
Ferritin & $510.00(266.50-886.75)$ & $548.5(268-1045.5)$ & $510(266.50-886.75)$ & 0.726 \\
Interleukin-6 & $41.64(22.77-75.45)$ & $58.16(26.8-153.15)$ & $41.17(22.52-74.82)$ & 0.145 \\
\hline
\end{tabular}

Bold values indicate statistically significant

$I Q R$ interquartile range; Leucocytes $(\times 10 \mathrm{E} 9 / \mathrm{L})$; ANC Absolute Neutrophil Count $(\times 10 \mathrm{E} 9 / \mathrm{L})$; ALC Absolute Lymphocyte Count $(\times 10 \mathrm{E} 9 / \mathrm{L})$; Fibrinogen $(\mathrm{g} / \mathrm{L})$; D-dimer $(\mathrm{ng} / \mathrm{mL}) ; L D H$ lactate dehydrogenase (UI/L); CRP C-reactive Protein (mg/dL); Ferritin (ng/mL); IL-6 Interleukin-6 (pg/mL)

*Data are expressed as median and IQR anti-coagulation was based on low molecular weight heparin, $40 \mathrm{mg}$ administered subcutaneously every day.
Eighty-eight patients (14.0\%) required admission in intensive care units (ICU) of whom sixty-five (73.9\%) required invasive mechanical ventilation (IMV). In those patients 
Table 4 Radiological findings from patients discharged after hospitalization for SARS-CoV-2 infection

\begin{tabular}{lllll}
\hline & Global $(N=629)$ & Readmitted $(N=34)$ & $\begin{array}{l}\text { Non-readmitted } \\
(N=595)\end{array}$ & $P$ value \\
\hline $\begin{array}{l}\text { Radiological abnor- } \\
\text { mality }\end{array}$ & $534(84.9)$ & $20(58.8)$ & $514(86.4)$ & $<\mathbf{0 . 0 0 1}$ \\
$\begin{array}{l}\text { Bilateral affectation } \\
\text { Extension }>33 \%\end{array}$ & $379(71)$ & $17(85)$ & $362(70.4)$ & 0.159 \\
\hline
\end{tabular}

Bold values indicate statistically significant

*Data are expressed as numbers and percentages unless otherwise indicated

Table 5 Treatment during the admission

\begin{tabular}{|c|c|c|c|c|}
\hline & Global $(N=629)$ & Readmitted $(N=34)$ & Non-readmitted $(N=595)$ & $P$ value \\
\hline Corticosteroids & $72(11.4)$ & $10(29.4)$ & $62(10.4)$ & 0.001 \\
\hline Immunotherapy & $111(17.6)$ & $9(26.5)$ & $102(17.1)$ & 0.165 \\
\hline Anticoagulation & & & & $<0.001$ \\
\hline $\begin{array}{l}\text { None } \\
\text { Prophylactic dosage } \\
\text { Full dosage }\end{array}$ & $\begin{array}{l}137(21.8) \\
432(68.8) \\
59(9.4)\end{array}$ & $\begin{array}{l}6(18.2) \\
16(48.5) \\
11(33 .)\end{array}$ & $\begin{array}{l}131(22.0) \\
416(69.9) \\
48(8.1)\end{array}$ & \\
\hline Admission in ICU & $88(14)$ & $8(23.5)$ & $80(13.4)$ & 0.099 \\
\hline Days in ICU (median, IQR) & $15(8-28)$ & $19(11-29)$ & $15(8-27)$ & 0.486 \\
\hline HFNC & $80(12.7)$ & $7(20.6)$ & $73(12.3)$ & 0.157 \\
\hline NIMV & $13(2.1)$ & $1(2.9)$ & $12(2)$ & 0.518 \\
\hline IMV & $65(10.3)$ & $6(17.6)$ & $59(9.9)$ & 0.150 \\
\hline Days with IMV (median, IQR) & $18(10-22)$ & $20(15-25)$ & $15(8-22)$ & 0.509 \\
\hline ECMO & $3(0.5)$ & $0(0)$ & $3(0.5)$ & 1.000 \\
\hline Maximum FiO2 needed (median, IQR) & $0.26(0.21-0.31)$ & $0.26(0.21-0.8)$ & $0.26(0.21-0.31)$ & 0.148 \\
\hline $\mathrm{FiO} 21$ & $35(5.6)$ & $3(8.8)$ & $32(5.4)$ & 0.427 \\
\hline Cytokine absorption & $3(0.5)$ & $1(2.9)$ & $2(0.3)$ & 0.154 \\
\hline Vasoactive support & $51(8.1)$ & $6(17.6)$ & 45 (7.6) & $\mathbf{0 . 0 3 6}$ \\
\hline Dialysis & $9(1.4)$ & $1(2.9)$ & $8(1.3)$ & 0.396 \\
\hline
\end{tabular}

Bold values indicate statistically significant

ICU Intensive Care Unit; IQR Intercuartilic range; HFNC High Flow Nasal Cannula; NIMV Non-Invasive Mechanical Ventilation; IMV Invasive Mechanical Ventilation; ECMO Extracorporeal Membrane Oxygenation; FiO2 Fraction of Inspired Oxygen

*Data are expressed as numbers and percentages unless otherwise indicated

who required admission in ICU, the median days of stay were 19 in readmitted patients and 15 in non-readmitted; for mechanical ventilation were 20 and 15 days, respectively. No statistically significant differences were found between both groups.

The readmitted group required more respiratory support. This support consisted of high-flow nasal cannula (20.6\% versus $12.3 \%)$, non-invasive mechanical ventilation $(2.9 \%$ versus $2 \%)$, mechanical ventilation $(17.6 \%$ versus $9.9 \%)$ and fraction of inspired oxygen ( $\mathrm{FiO} 2)$ of $100 \%$ administered with any device (8.8\% versus 5.4\%). Additionally, this group required vasoactive support (17.6\% versus $7.6 \%$ ) and dialysis $(2.9 \%$ versus $1.3 \%)$ in a greater percentage than the nonreadmitted group. In spite of that, we only found statistically significant differences among the need for vasoactive support $(p=0.036)$.

\section{Complications developed during admission}

Several complications were described in hospitalized patients: ARDS (14.9\%), respiratory failure (28.6\%), heart failure $(2.1 \%)$, secondary infection $(10.3 \%)$, arrhythmia (5.6\%) or PTE (1.6\%) among others. Clinical complications are detailed in Table 6.

No statistical differences were found between patients who developed or did not ARDS or respiratory failure. Acute kidney failure and liver dysfunction were described in 5.7 and $4.0 \%$ of the patients, respectively. None of the 
Table 6 Complications developed during the admission $\begin{array}{lll}\text { Global }(N=629) & \text { Readmitted }(N=34) & \begin{array}{l}\text { Non-readmit- } \\ \text { ted }(N=595)\end{array}\end{array} \quad P$ value

\begin{tabular}{lccrr}
\hline ARDS & $94(14.9)$ & $9(26.5)$ & $85(14.3)$ & 0.053 \\
Respiratory failure & $180(28.6)$ & $13(38.2)$ & $167(28.1)$ & 0.202 \\
Acute heart failure & $13(2.1)$ & $3(8.8)$ & $10(1.7)$ & $\mathbf{0 . 0 2 8}$ \\
Septic shock & $11(1.7)$ & $1(2.9)$ & $10(1.7)$ & 0.460 \\
Acute kidney failure & $36(5.7)$ & $3(8.8)$ & $33(5.5)$ & 0.435 \\
Secondary infection & $65(10.3)$ & $10(29.4)$ & $55(9.2)$ & $<\mathbf{0 . 0 0 1}$ \\
Seizures & $1(0.2)$ & $0(0)$ & $1(0.2)$ & 1.000 \\
Stroke & $1(0.2)$ & $0(0)$ & $1(0.2)$ & 1.000 \\
Myocarditis & $1(0.2)$ & $0(0)$ & $1(0.2)$ & 1.000 \\
Pericarditis & $2(0.3)$ & $1(2.9)$ & $1(0.2)$ & 0.105 \\
Endocarditis & $1(0.2)$ & $1(2.9)$ & $0(0)$ & 0.054 \\
Arrhythmia & $15(2.4)$ & $2(5.9)$ & $13(2.2)$ & 0.192 \\
PTE & $10(1.6)$ & $4(11.8)$ & $6(1)$ & $\mathbf{0 . 0 0 1}$ \\
DVP & $13(2.1)$ & $2(5.9)$ & $11(1.8)$ & 0.152 \\
Anemia, required transfusions & $14(2.2)$ & $2(5.9)$ & $12(2)$ & 0.172 \\
Liver dysfunction & $25(4)$ & $1(2.9)$ & $24(4)$ & 1.000 \\
\hline
\end{tabular}

Bold values indicate statistically significant

ARDS Acute Respiratory Distress Syndrome; PTE Pulmonary Thromboembolism; DVP Deep-Vein Thrombosis

*Data are expressed as numbers and percentages unless otherwise indicated

\begin{tabular}{|c|c|c|c|c|c|c|}
\hline \multirow[t]{2}{*}{ Variable } & \multicolumn{3}{|c|}{ Univariate analysis } & \multicolumn{3}{|c|}{ Multivariate analysis } \\
\hline & $p$ & OR & $95 \% \mathrm{CI}$ & $p$ & OR & $95 \% \mathrm{CI}$ \\
\hline PTE & $<0.001$ & 13.09 & $3.51-48.86$ & 0.001 & 11.59 & $2.89-46.48$ \\
\hline Secondary infection & $<0.001$ & 4.09 & $1.86-9.00$ & & & \\
\hline Length of stay $>13$ days & $<0.001$ & 4.07 & $1.94-8.51$ & 0.015 & 2.72 & $1.21-6.12$ \\
\hline Treatment with corticoids & 0.001 & 3.58 & $1.64-7.84$ & 0.049 & 2.39 & $1.01-5.70$ \\
\hline Prior diagnosis of heart failure & 0.004 & 4.71 & $1.66-13.39$ & 0.013 & 4.09 & $1.35-12.46$ \\
\hline $\mathrm{CCI}>3$ & 0.005 & 2.77 & $1.37-5.61$ & & & \\
\hline Drinking habit & 0.007 & 4.10 & $1.46-11.53$ & & & \\
\hline Radiologic affectation $>33 \%$ & 0.010 & 3.26 & $1.32-8.03$ & & & \\
\hline ALC $<0.789 \times 109 / \mathrm{L}$ & 0.010 & 2.52 & $1.25-5.06$ & & & \\
\hline Acute heart failure (complication) & 0.011 & 5.66 & $1.48-21.62$ & & & \\
\hline Smoking habit & 0.015 & 2.38 & $1.18-4.81$ & & & \\
\hline D-dimer $>233 \mathrm{ng} / \mathrm{mL}$ & 0.015 & 3.46 & $1.28-9.50$ & & & \\
\hline Prior diagnosis of cancer & 0.030 & 2.64 & $1.10-6.36$ & & & \\
\hline Need of vasoactive support & 0.043 & 2.62 & $1.03-6.66$ & & & \\
\hline Male gender & 0.045 & 2.13 & $1.02-4.44$ & & & \\
\hline ADRS & 0.058 & 2.16 & $0.98-4.79$ & & & \\
\hline Diabetes mellitus & 0.084 & 1.97 & $0.91-4.24$ & & & \\
\hline Admission in ICU & 0.105 & 1.98 & $0.87-4.53$ & & & \\
\hline
\end{tabular}

OR Odds Ratio; CI Confidence Interval; PTE Pulmonary thromboembolism; CCI Charlson Comorbidity Index; ALC Absolute Lymphocyte Count; ADRS Acute Distress Respiratory Syndrome; ICU Intensive Care Unit
Table 7 Univariate and multivariate analysis of risk factors associated with readmission after hospitalization for SARS-CoV-2 infection 
complications mentioned above showed statistically significant differences nor the presence of septic shock. In contrast, those patients who underwent a secondary infection $(p<0.001)$, acute heart failure $(p=0.028)$ or PTE $(p=0.001)$ had higher rates of readmission.

\section{Risk factors associated with readmission}

Table 7 summarizes the results of both univariate and multivariate analysis of risk factors.

Although we found many different risk factors associated with readmission, only four of them kept their significance in the multivariate analysis. These risk factors were prior diagnosis of heart failure (OR 4.09; 95\% CI 1.35-12.46; $p=0.013$ ), length of stay $>13$ days (OR $2.39 ; 95 \%$ CI $1.21-6.12 ; p=0.015)$, treatment with corticoids (OR 2.39; 95\% CI 1.01-5.7; $p=0.049$ ) and developing PTE (OR 11.59; $95 \%$ CI $2.89-46.48 ; p=0.001)$.

\section{Discussion}

In this retrospective cohort study of 870 patients admitted to a tertiary care hospital in Spain, we identified an overall 60 -day readmission cumulative incidence of $5.4 \%$. Of those readmitted, half of the patients were readmitted within two weeks of discharge. We found that readmission was associated with factors related to a more severe COVID-19, such as a longer hospital stay, the need for corticosteroids or the development of PTE, or the presence of previous underlying disease such as cardiac insufficiency.

On the one hand, the virus principally targets cells which express the angiotensin-converting enzyme 2 (ACE2), such as airway epithelial cells, alveolar epithelial cells, vascular endothelial cells and macrophages in the lung. This receptor has been shown to regulate the renin-angiotensin system (RAS). The infection appears to reduce ACE2 expression and could result in a dysfunction of the RAS, which influences blood pressure and fluid/electrolyte balance and enhances inflammation and vascular permeability in the airways [12]. As a result, a pattern of hematologic, biochemical, inflammatory and immune biomarker abnormalities has been identified in patients with severe disease and seems to correlate with the prognosis [13]. Despite that, we did not find lymphopenia to maintain independent statistical impact in the multivariate analysis. Similar to other viral infections, such as measles, respiratory syncytial virus, SARS-CoV or MERS-CoV, current reports emphasize the presence of lymphopenia in severe COVID-19 cases. The mechanism of lymphopenia remains incompletely understood but it may be secondary to an increased apoptosis in T cells and pulmonary recruitment of immune cells [14].
In addition, the above-mentioned pro-inflammatory state may play a role for the decompensation of a stable disease. It has been proposed that neuro-hormonal activation, inflammatory activation and oxidative stress may have a primary role in the progression of a stable/chronic disease, mostly in acute heart failure or decompensated cirrhosis $[15,16]$. These complications may not be associated only with SARSCoV-2 infection but also with its indirect medical effects on the healthcare system [17]. In our cohort, more than thirty percent of the readmissions were due to a decompensation of a baseline disease. Among these, thirty percent stemmed from acute heart failure.

On the other hand, ACE2 receptors are also expressed in endothelial cells and may be dysregulated by SARS-CoV-2 infection. This may result in vasoconstriction of microcirculation, organ ischaemia and a pro-coagulant state [18]. Cytokine release also contributes in a pro-coagulant state due to an activation of platelet activity and inducing tissue factor $[19,20]$. All of it leads to an increased incidence of venous thromboembolism in patients with SARS-CoV-2 pneumonia and poorer prognosis [21]. Low molecular weight heparin (LMWH) has an anticoagulant effect and reduces the pro-inflammatory cytokines release in patients with COVID-19 infection [22]. Our findings suggest that PTE is a major cause of readmission and the administration of prophylactic anti-coagulation in hospitalized patients might lead to lower readmission rates.

Current recommendations on treatment are based on the use of dexamethasone. The benefit of steroid treatment has only been demonstrated in patients requiring oxygen therapy [23]. Despite that, the optimal dose and duration of treatment remain unclear. Further studies are required in this field. The anti-inflammatory properties of steroids seem to reduce hospital stay length, progression to needing IVM and mortality. Conversely, its immunosuppressant effect can lead to an increased risk of bacterial superinfection or they may increase the risk of developing other systemic complications, such as cardiovascular events [24]. Therefore, treatment with corticoids may play a role in those readmissions due to infectious complications and in baseline diseases decompensations. In our study, we did not obtain any evidence about the role of low absolute lymphocyte count and/or corticosteroid administration in fungal invasive infections. In fact, we did not find any fungal infections in the readmitted patients. What we observed was that, in several patients who were admitted to the ICU, strains of Aspergillus spp. were isolated in tracheal samples. Otherwise, in none of the cases, an invasive fungal infection was described.

Finally, despite several respiratory complications, such as ARDS or respiratory failure, are related to disease severity, none of them had a statistical impact on the readmission rate. A similar association is highlighted in Townsend et al. [25], where they describe a persistence of ill health 
and fatigue following SARS-CoV-2 infection independent of initial severity of infection.

Similar to an observational cohort study performed by Drewett et al. [26], we found that a longer length of stay during index admission was significantly associated with readmission. This may be associated with a more severe infection and complications developed during hospitalization, which would imply a longer admission.

Of note, only one admission more than 15 days after the discharge was related to a respiratory event. Similar findings are reflected in Sulaiman et al. [11] where readmission at 14 days after discharge is mainly due to respiratory distress. Infectious complications were more frequent between 10 and 30 days after discharge. As suggested in previous studies, patients with comorbidities can be at a high risk of decompensation of their chronic diseases for at least two months after discharge. This suggests that an enhanced follow-up for patients with a high comorbidity burden should be warranted to prevent possible readmissions and further complications.

In a retrospective observational study performed by Yeo et al. [7], readmission cumulative incidence at 30 days after discharge was $4.5 \%$ and mainly due to hypoxic respiratory failure, PTE and sepsis. Donnelly et al. [10] analyzed readmission among 2179 patients; $19.9 \%$ of them had to be readmitted, mostly due to COVID-19, sepsis, pneumonia and heart failure. Of note, in this study, they found that COVID-19 survivors had lower rates of 60-day readmission than matched survivors of pneumonia and heart failure. Finally, in another study of 106,543 patients, Lavery et al. [27] found an incidence of $9 \%$ within 2 months, mainly due to complications of underlying conditions. These findings are similar to ours but cannot be compared due to different study designs. Further studies are necessary to elucidate these results.

\section{Limitations}

Our study has several limitations. First, it is a single-hospital-based study, which reflects only the clinical practice we applied. It also might underestimate the readmission rate by not capturing readmissions to other institutions since the study population only included patients within Vall d'Hebron University Hospital, Barcelona, Spain.

Second, we used a retrospective database and the data were collected from the electronic health record database. This could have led to missing information in some patients.

Finally, the clinical practice in COVID-19 is permanently evolving as more evidence is blooming. Patients receiving different treatment options may display varying readmission rates.

\section{Conclusion}

In our study cohort, we had a readmission cumulative incidence of $5.4 \%$. The most common causes for readmission were respiratory worsening or decompensation of an underlying disease. Independent risk factors for readmission were previous diagnosis of cardiac insufficiency, increased length of stay, developing PTE and treatment with corticoids. Our findings can help focusing follow-up after discharge in those patients who are more likely to require re-hospitalization.

Funding This research did not receive any specific grant from funding agencies in the public, commercial, or not-for-profit sectors.

\section{Declarations}

Conflict of interest The authors declare they have no conflict of interest.

Ethics approval The institutional review board provided ethical clearance (local review board code number: PR(AG)183/2020). Patients were asked for oral consent. The institutional review board granted an informed consent waiver if patients were unable to give oral consent. Written consent was waived because of the crisis context and concerns about safety when introducing a physical support for the consent in the isolation areas.

\section{References}

1. European Centre for Disease Prevention and Control (ECDC). Available at: https://www.ecdc.europa.eu/en. Accessed 27 June 2021.

2. Guan WJ, Ni ZY, Hu Y, Liang WH, Ou CQ, He JX, et al. Clinical characteristics of coronavirus disease 2019 in China. N Engl J Med. 2020;382:1708-20. https://doi.org/10.1056/NEJMoa2002 032.

3. Yang X, Yu Y, Xu J, Shu H, Xia J, Liu H, et al. Clinical course and outcomes of critically ill patients with SARS-CoV-2 pneumonia in Wuhan, China: a single-centered, retrospective, observational study. Lancet Respir Med. 2020;8:475-81. https://doi.org/10. 1016/S2213-2600(20)30079-5.

4. Zhang JJ, Dong X, Cao YY, Yuan YD, Yang YB, Yan YQ, et al. Clinical characteristics of 140 patients infected with SARS-CoV-2 in Wuhan, China. Allergy. 2020;75:1730-4. https://doi.org/10. 1111/all.14238.

5. Zhou F, Yu T, Du R, Fan G, Liu Y, Liu Z, et al. Clinical course and risk factors for mortality of adult inpatients with COVID19 in Wuhan, China: a retrospective cohort study. Lancet. 2020;395:1054-62. https://doi.org/10.1016/S0140-6736(20) 30566-3.

6. Ejaz H, Alsrhani A, Zafar A, Javed H, Junaid K, Abdalla AE, et al. COVID-19 and comorbidities: deleterious impact on infected patients. J Infect Public Health. 2020;13:1833-9. https://doi.org/ 10.1016/j.jiph.2020.07.014.

7. Yeo I, Baek S, Kim J, Elshakh H, Voronina A, Lou MS, et al. Assessment of thirty-day readmission rate, timing, causes and predictors after hospitalization with COVID-19. J Intern Med. 2021. https://doi.org/10.1111/joim.13241 (Online ahead of print). 
8. Saab FG, Chiang JN, Brook R, Adamson PC, Fulcher JA, Halperin $\mathrm{E}$, et al. Discharge clinical characteristics and post-discharge events in patients with severe COVID-19: a descriptive case series. J Gen Intern Med. 2021. https://doi.org/10.1007/s11606020-06494-7 (Online ahead of print).

9. Casas-Rojo JM, Anton-Santos JM, Millan-Nunez-Cortes J, Lumbreras-Bermejo C, Ramos-Rincon JM, Roy-Vallejo E, et al. Clinical characteristics of patients hospitalized with COVID-19 in Spain: results from the SEMI-COVID-19 registry. Rev Clin Esp. 2020;220:480-94. https://doi.org/10.1016/j.rce.2020.07.003.

10. Donnelly JP, Wang XQ, Iwashyna TJ, Prescott HC. Readmission and death after initial hospital discharge among patients with COVID-19 in a large multihospital system. JAMA. 2021;325:304 6. https://doi.org/10.1001/jama.2020.21465.

11. Somani SS, Richter F, Fuster V, De Freitas JK, Naik N, Sigel K, et al. Characterization of patients who return to hospital following discharge from hospitalization for COVID-19. J Gen Intern Med. 2020;35:2838-44. https://doi.org/10.1007/s11606-020-06120-6.

12. Tay MZ, Poh CM, Renia L, MacAry PA, Ng LFP. The trinity of COVID-19: immunity, inflammation and intervention. Nat Rev Immunol. 2020;20:363-74. https://doi.org/10.1038/ s41577-020-0311-8.

13. Ponti G, Maccaferri M, Ruini C, Tomasi A, Ozben T. Biomarkers associated with COVID-19 disease progression. Crit Rev Clin Lab Sci. 2020;57:389-99. https://doi.org/10.1080/10408363.2020. 1770685.

14. Chen Z, John WE. T cell responses in patients with COVID-19. Nat Rev Immunol. 2020;20:529-36. https://doi.org/10.1038/ s41577-020-0402-6.

15. Farmakis D, Parissis J, Lekakis J, Filippatos G. Acute heart failure: epidemiology, risk factors, and prevention. Rev Esp Cardiol (Engl Ed). 2015;68:245-8. https://doi.org/10.1016/j.rec.2014.11. 004

16. Bajaj JS, Garcia-Tsao G, Wong F, Biggins SW, Kamath PS, McGeorge $\mathrm{S}$, et al. Cirrhosis is associated with high mortality and readmissions over 90 days regardless of COVID-19: a multicenter cohort. Liver Transpl. 2021. https://doi.org/10.1002/lt. 25981 (Online ahead of print).

17. Ahmed T, Lodhi SH, Kapadia S, Shah GV. Community and healthcare system-related factors feeding the phenomenon of evading medical attention for time-dependent emergencies during COVID-19 crisis. BMJ Case Rep. 2020;13:e237817. https:// doi.org/10.1136/bcr-2020-237817.
18. Varga Z, Flammer AJ, Steiger P, Haberecker M, Andermatt R, Zinkernagel AS, et al. Endothelial cell infection and endotheliitis in COVID-19. Lancet. 2020;395:1417-8. https://doi.org/10.1016/ S0140-6736(20)30937-5.

19. Van der Poll T, Levi M. Crosstalk between inflammation and coagulation: the lessons of sepsis. Curr Vasc Pharmacol. 2012;10:632-8. https://doi.org/10.2174/157016112801784549.

20. Opal SM. Interactions between coagulation and inflammation. Scand J Infect Dis. 2003;35:545-54. https://doi.org/10.1080/ 00365540310015638

21. Cui S, Chen S, Li X, Liu S, Wang F. Prevalence of venous thromboembolism in patients with severe novel coronavirus pneumonia. J Thromb Haemost. 2020;18:1421-4. https://doi.org/10.1111/jth. 14830.

22. Miesbach W, Makris M. COVID-19: coagulopathy, risk of thrombosis, and the rationale for anticoagulation. Clin Appl Thromb Hemost. 2020;26:1076029620938149. https://doi.org/10.1177/ 1076029620938149 .

23. The WHO Rapid Evidence Appraisal for COVID-19 Therapies (REACT) Working Group. Association between administration of systemic corticosteroids and mortality among critically ill patients with COVID-19: a meta-analysis. JAMA. 2020;324:1330-41. https://doi.org/10.1001/jama.2020.17023.

24. Mattos-Silva P, Felix NS, Silva PL, Robba C, Battaglini D, Pelosi $\mathrm{P}$, et al. Pros and cons of corticosteroid therapy for COVID-19 patients. Respir Physiol Neurobiol. 2020;280: 103492. https://doi. org/10.1016/j.resp.2020.103492.

25. Townsend L, Dowds J, O’Brien K, Sheill G, Dyer AH, O'Kelly $\mathrm{B}$, et al. Persistent poor health post-COVID-19 is not associated with respiratory complications or initial disease severity. Ann Am Thorac Soc. 2021. https://doi.org/10.1513/AnnalsATS.202009$1175 \mathrm{OC}$ (Online ahead of print).

26. Drewett GP, Chan RK, Jones N, Wimaleswaran H, Howard ME, McDonald CF, et al. Risk factors for readmission following inpatient management of COVID-19 in a low-prevalence setting. Intern Med J. 2021;51:821-3.

27. Lavery AM, Preston LE, Ko JY, Chevinsky JR, DeSisto CL, Pennington AF, et al. Characteristics of hospitalized COVID-19 patients discharged and experiencing same-hospital readmission-United States, March-August 2020. MMWR Morb Mortal Wkly Rep. 2020;69:1695-9. https://doi.org/10.15585/mmwr. mm6945e2. 